\title{
Antigen-mediated regulation in monoclonal gammopathies and myeloma
}

\author{
Shiny Nair, Joel Sng, ${ }^{2}$ Chandra Sekhar Boddupalli, ${ }^{1}$ Anja Seckinger, ${ }^{3}$ Marta Chesi, ${ }^{4}$ \\ Mariateresa Fulciniti, ${ }^{5}$ Lin Zhang, ${ }^{1}$ Navin Rauniyar, ${ }^{6}$ Michael Lopez, ${ }^{5}$ Natalia Neparidze, ${ }^{1}$ \\ Terri Parker, ${ }^{1}$ Nikhil C. Munshi, ${ }^{5}$ Rachael Sexton, ${ }^{7}$ Bart Barlogie, ${ }^{8}$ Robert Orlowski, ${ }^{9}$ Leif Bergsagel, ${ }^{4}$ \\ Dirk Hose, ${ }^{3}$ Richard A. Flavell, ${ }^{2}$ Pramod K. Mistry, ${ }^{1}$ Eric Meffre, ${ }^{2}$ and Madhav V. Dhodapkar ${ }^{1,2}$ \\ 1Department of Medicine and ${ }^{2}$ Immunobiology, Yale University, New Haven, Connecticut, USA. ${ }^{3}$ Labor für \\ Myelomforschung, Medizinische Klinik V, Universitätsklinikum Heidelberg, Heidelberg, Germany. ${ }^{4}$ Mayo Clinic, Scottsdale,

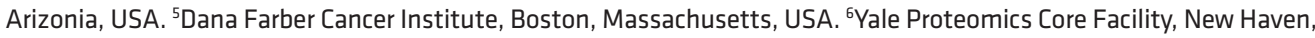 \\ Connecticut, USA. ${ }^{7}$ Cancer Research and Biostatistics, Southwest Oncology Group (SWOG), Seattle, Washington, USA. \\ ${ }^{8}$ Mount Sinai Medical Center, New York, New York, USA. ${ }^{9}$ MD Anderson Cancer Center, Houston, Texas.
}

A role for antigen-driven stimulation has been proposed in the pathogenesis of monoclonal gammopathy of undetermined significance (MCUS) and multiple myeloma (MM) based largely on the binding properties of monoclonal Ig. However, insights into antigen binding to clonal $B$ cell receptors and in vivo responsiveness of the malignant clone to antigenmediated stimulation are needed to understand the role of antigenic stimulation in tumor growth. Lysolipid-reactive clonal Ig were detected in Gaucher disease (CD) and some sporadic gammopathies. Here, we show that recombinant Ig (rlg) cloned from sort-purified single tumor cells from lipid-reactive sporadic and CD-associated gammopathy specifically bound lysolipids. Liposome sedimentation and binding assays confirmed specific interaction of lipid-reactive monoclonal Ig with lysolipids. The clonal nature of lysolipid-binding Ig was validated by protein sequencing. Gene expression profiling and cytogenetic analyses from 2 patient cohorts showed enrichment of nonhyperdiploid tumors in lipid-reactive patients. In vivo antigen-mediated stimulation led to an increase in clonal Ig and plasma cells (PCs) in CD gammopathy and also reactivated previously suppressed antigenically related nonclonal PCs. These data support a model wherein antigenic stimulation mediates an initial polyclonal phase, followed by evolution of monoclonal tumors enriched in nonhyperdiploid genomes, responsive to underlying antigen. Targeting underlying antigens may therefore prevent clinical MM.

Conflict of interest: The authors have declared that no conflict of interest exists.

Submitted: October 24, 2017 Accepted: March 16, 2018 Published: April १९, 2018

Reference information: JCI Insight. 2018;3(8):e98259. https://doi.org/10.1172/jci. insight.98259.

\section{Introduction}

Multiple myeloma (MM) and its precursor monoclonal gammopathy of undetermined significance (MGUS) are characterized by the growth of clonal plasma cells in the BM $(1,2)$. It is now well appreciated that genetic heterogeneity and genomic instability is a common feature of $\mathrm{MM}$, and much of this heterogeneity is established early, during MM pathogenesis (3-6). MM is classified into 2 broad subtypes based on cytogenetics (7). Tumors with hyperdiploid (HY) karyotypes account for nearly $50 \%$ of the patients and typically have trisomies of odd-numbered chromosomes 3, 5, 7, 9, 11, 15, 19, and 21 (7). In contrast, non-HY MM is characterized by translocations involving the immunoglobulin heavy chain (IGH) locus at 14q32 (8). Gene expression profiling (GEP) of purified tumor cells helped classify MM into 7 major genomic subtypes, which can also be detected in MGUS patients $(5,9)$. It is unknown whether these molecular subtypes differ in terms of their underlying pathogenic mechanisms.

A possible role of lipids in the pathogenesis of MM dates back to early studies with pristane-induced plasma cell tumors in mice (10). Risk of myeloma is markedly increased in patients with Gaucher disease (GD), an inherited lipid storage disorder $(11,12)$. Accumulation of glucosphingolipids (GSL) in GD leads to the activation of GSL-reactive NKT cells with T-follicular helper (Tfh) phenotype, which provide help for plasma cell differentiation and lipid-specific antibodies (13). In prior studies, we have shown that monoclonal Igs (mIg) in GD patients and some patients with sporadic MM in non-GD settings can bind GD-associated or related lysolipids (14). Pharmacologic reduction of antigenic lipids led to reduction in 
the risk of gammopathy in vivo in GD animal models $(14,15)$. These data support the hypothesis that lipid-mediated chronic immune activation underlies the development of monoclonal gammopathies in GD and a subset of sporadic MM.

We therefore sought to delineate the role of lipid-mediated chronic stimulation in the initiation and progression of MM. In this study, we address whether lysolipids bind to clonal B cell receptors and whether lipid reactivity occurs in specific molecular subtypes of MM. Finally, we investigated whether the malignant clone in lipid-reactive gammopathy (LRG) respond to antigenic stimulation in vivo.

\section{Results}

Igs cloned from clonal B cell receptors in human MM and GD-associated gammopathy bind lysolipids. We have previously shown, utilizing lipid-specific immunoblots, that clonal Igs in GD-associated monoclonal gammopathy (GD-MG) and a subset of patients with sporadic MM can bind lysolipids. In order to directly demonstrate that lipid-reactive Ig in patients with LRG is derived from clonal plasma cells, we cloned and expressed recombinant Ig (rIg) from single-cell-sorted $\mathrm{CD} 38^{+} \mathrm{CD} 138^{+}$tumor cells from patients with sporadic MM and GD-MG, as outlined in Methods and Supplemental Figure 1 (supplemental material available online with this article; https://doi.org/10.1172/jci.insight.98259DS1). Briefly, we performed single-cell sorting of $\mathrm{CD}_{3} 8^{+} \mathrm{CD} 138^{+}$plasma cells by flow cytometry (Supplemental Figure 2) from $4 \mathrm{MM}$ patients ( 2 with LRG and 2 with nonreactive gammopathy; NRG) and 1 GD patient with LRG. For each cell, the corresponding heavy and light chain variable regions were amplified by nested PCR and cloned into expression vectors, and recombinant monoclonal human antibodies were expressed in HEK293 cells. Clonality was confirmed based on sequence identity of $\operatorname{Ig}$ heavy $(\operatorname{IgH})$ and $\operatorname{Ig}$ light (IgL) chains as analyzed by IgBlast (https://www.ncbi.nlm.nih.gov/igblast/) or ImMunoGeneTics information system (IMGT; http://www.imgt.org/; Supplemental Table 1). This data also demonstrated the presence of somatic mutations in the heavy chain sequence of the cloned Ig genes (Supplemental Figure 3). rIg from patients with LRG, as well as GD, demonstrated glucosylsphingosine (GlcSph) reactivity, while no reactivity was observed in rIg from NRG patients (Figure 1A). Reactivity of rIg in this assay was comparable with that of naturally occurring clonal Ig (Figure 1B). Binding of purified clonal Ig from LRG patient (R1) to GlcSph was competitively inhibited by the $\mathrm{F}(\mathrm{ab})_{2}$ fragment of rIg from this patient, while no such competition was observed with the $\mathrm{F}(\mathrm{ab})_{2}$ fragment from an NRG patient (N1) (Figure 1C). Similarly, binding of purified Ig from the sera of GD-MG to GlcSph was competitively inhibited by the $\mathrm{F}(\mathrm{ab})_{2}$ fragment of $\mathrm{rIg}$ from this patient (Figure 1D). These data, therefore, confirm the clonal nature of lipid-reactive Ig in both sporadic MM and GD-MG and demonstrate the capacity of clonal B cell receptors to bind antigen.

Binding of mIg to GlcSph-containing liposomes and C18 silica beads. Our next objective was to assess interaction of clonal Igs to lysolipids using techniques that present the lysolipid closer to physiologic context and polarity. For this mIg, serum samples of LRG patients were first purified, and the purity of the mIg was confirmed by isoelectric focusing (IEF) and Western blot using specific heavy chain antibody (Supplemental Figure 4, A and B). Lipid reactivity of the mIg was also verified using lipid-specific immunoblotting (Supplemental Figure 4C). In prior studies, we had utilized sphingosine beads as a source of antigen for enrichment and depletion of M spike from LRG plasma (14). The capacity of these beads to bind clonal Ig based on depletion of clonal Igs, as well as elution of bound lipid as reported previously (14), was independently verified in 2 separate labs (M. Chesi/L. Bergsagel and M. Fulciniti/N. Munshi; Supplemental Figure 5). However, since the sphingosine beads have a low carrying capacity (10 $\mathrm{nM}$ of bound lipid/ml of beads) and sphingosine is not the complete antigen, we utilized GlcSph-loaded liposomes, which also have the advantage of presenting lipid antigen in a more physiological context (i.e., in a lipid bilayer) to directly test lipid-binding properties of clonal Ig. Size of GlcSph-loaded liposomes and the concentration of GlcSph loaded on liposomes were verified by dynamic light scattering (DLS) and mass spectrometry (MS), respectively (Supplemental Figure 6). In pilot studies, we observed that liposomes made exclusively with GlcSph, owing to a single carbon chain, were not stable and led to aggregate formation (data not shown). Therefore, we utilized a combination of cholesterol and phosphatidylcholine (PC) with varying concentration of GlcSph for preparing GlcSph-containing liposomes. These liposomes demonstrated stability over 3 weeks at $4^{\circ} \mathrm{C}$. Liposomes containing cholesterol and $\mathrm{PC}$ without added GlcSph were used as control to evaluate background binding. Liposome sedimentation assay was used to measure binding of purified Igs from lipid-reactive patients to GlcSph-containing liposomes. Both control and GlcSph liposomes containing increasing concentrations of GlcSph were incubated with purified mIgs 
A
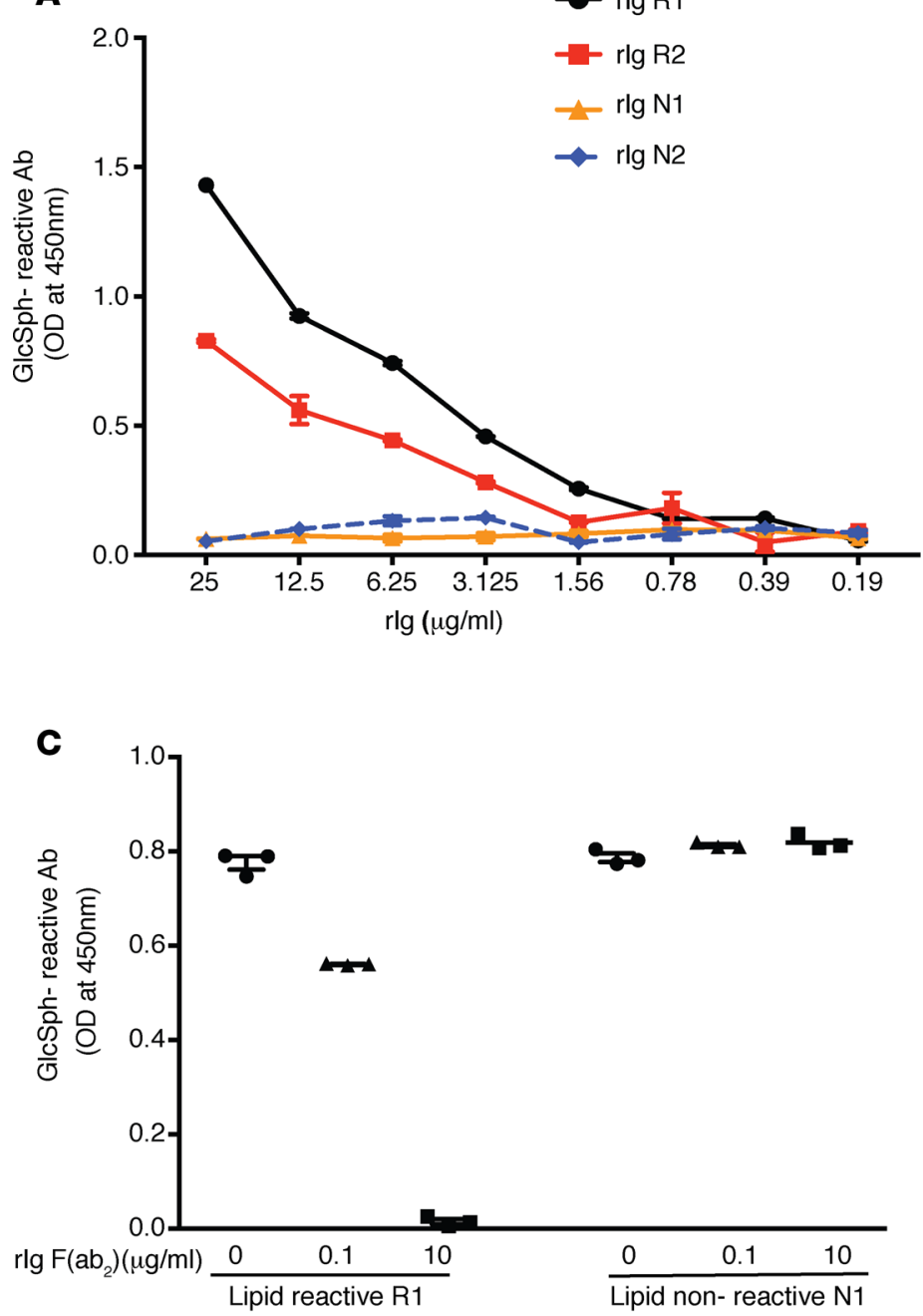

B

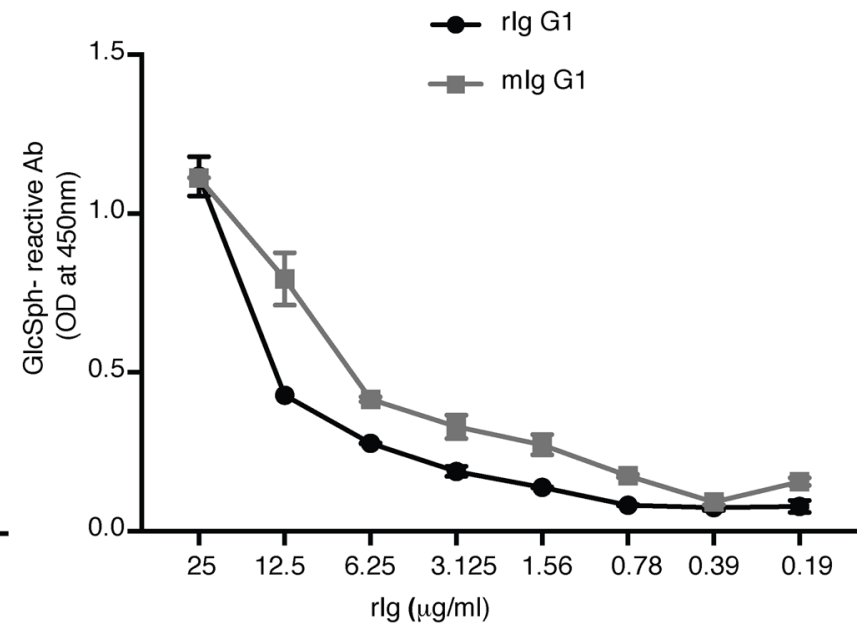

D

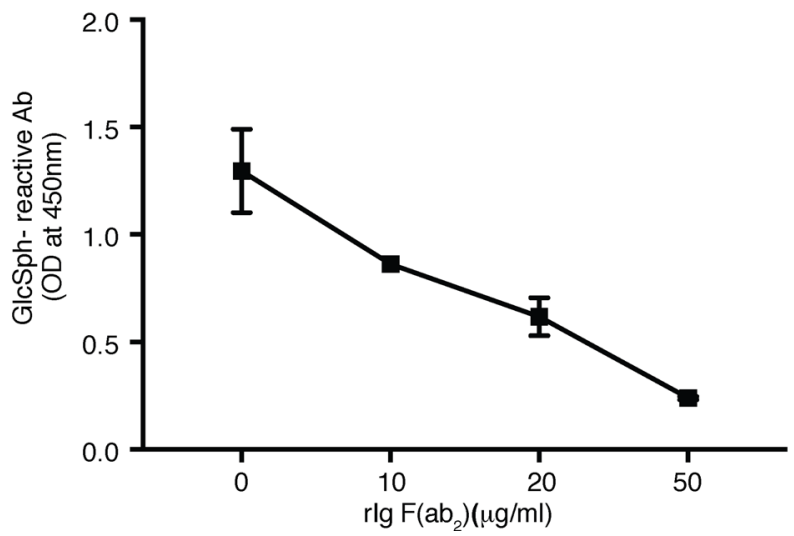

Figure 1. Reactivity and specificity of recombinant monoclonal Ig binding to GlcSph. (A) Recombinant Ig (rlg) cloned from single-cell sorted tumors $\left(\right.$ CD38 ${ }^{+}$CD138 $)$from lipid-reactive patients $(n=2)$ R1 and R2 showed GlcSph reactivity, while rlg cloned from lipid nonreactive patients $(n=2)$ N1 and N2 showed no reactivity in GIcSph-specific ELISA. (B) rlg cloned from single sorted plasma cells from lipid reactive Gaucher disease patient with monoclonal gammopathy (GD-MG; G1) show similar GlcSph reactivity as monoclonal lg (mlg) purified from the patient's sera. (C) Specificity of cloned rlg-derived F(ab) to bind GlcSph was assessed by competition ELISA with corresponding serum-purified mlg. GlcSph-coated well were incubated with increasing concentration of purified recombinant $\mathrm{F}(\mathrm{ab})_{2}$ from lipid reactive (R1) and non-lipid reactive (N1) patients. GlcSph binding of purified lg (25 $\left.\mu \mathrm{g} / \mathrm{ml}\right)$ from sera of patient R1 was inhibited in the presence of corresponding $\mathrm{F}(\mathrm{ab})_{2}$ from R1 but not N1. (D) GlcSph reactivity of monoclonal lg ( $\left.25 \mu \mathrm{g} / \mathrm{ml}\right) \mathrm{purified} \mathrm{from} \mathrm{the}$ CD-MG patient's (G1) sera was competitively inhibited by increasing concentration of corresponding rlg-derived $F(a b)$. Data represent mean \pm SEM.

from sporadic MM patients with LRG, and partitioning of mIgs into the GlcSph liposome pellet was assessed by immunoblot. Purified mIg bound to GlcSph-bearing liposomes in a dose-dependent manner, whereas binding to control liposomes was negligible (Figure 2A). Similar results were obtained in experiments performed with purified Igs from a GD patient with GlcSph (Figure 2B).

We next developed flow-based assays to measure the interactions between mIg and GlcSph liposomes. Fluorescently labeled $\mathrm{mIg}$ was incubated with both control and GlcSph-containing liposomes with varying concentration of GlcSph, and the binding of mIg to liposomes was analyzed by flow cytometry. As observed in the histogram, clonal Ig bound to GlcSph liposomes in a dose-dependent manner (Figure 2C). In addition to liposome binding, GlcSph binding of purified Igs from sporadic MM with LRG was also tested using GlcSph-coated silica beads that were end capped with hydrophobic $\mathrm{C} 18$ alkyl groups on their surface. Lipid-coated C18 silica beads have been used in prior studies to study lipid-protein interactions (16). GlcSph containing C18 silica beads had a similar composition as that of GlcSph-containing liposome, while control C18 silica beads had only the backbone lipids cholesterol and PC. Fluorescently labeled purified mIg from a lipid-reactive patient was incubated with either control or GlcSph-containing liposomes and the binding was assessed by 
A
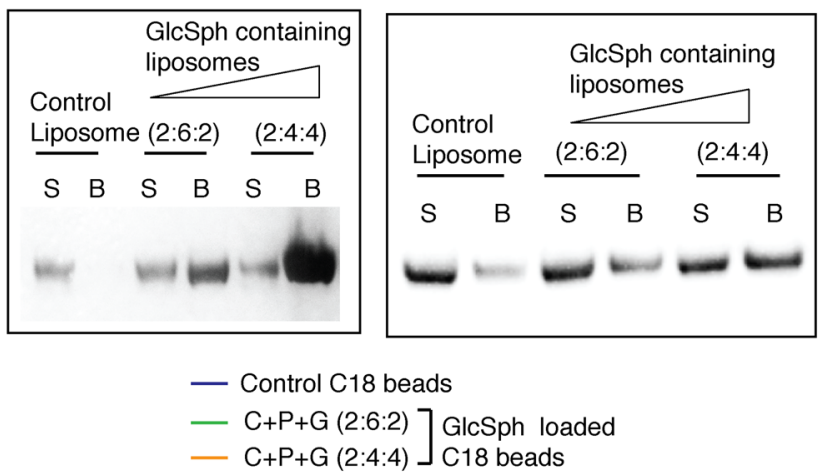

D Binding of $\mathrm{mlg}$ to GlcSph loaded $\mathrm{C} 18$ beads

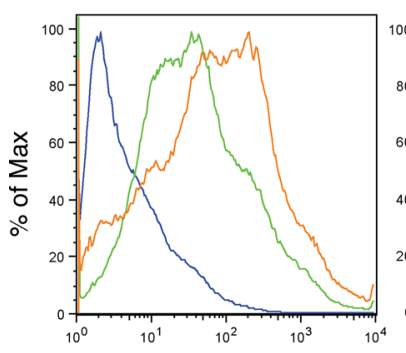

FITC labeled mlg

\section{E}

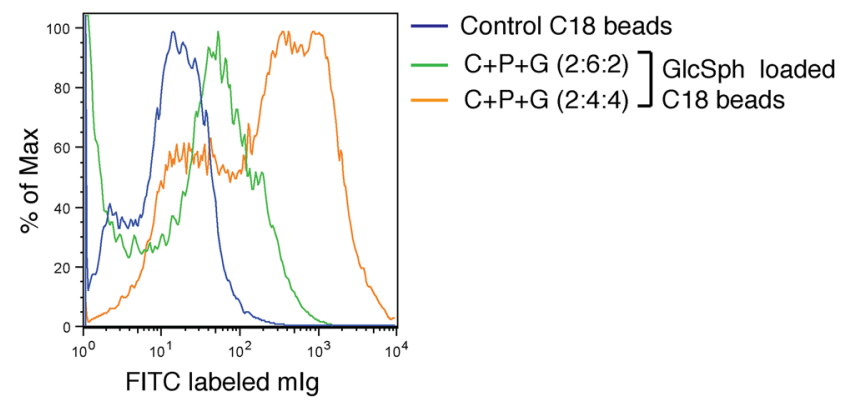

C

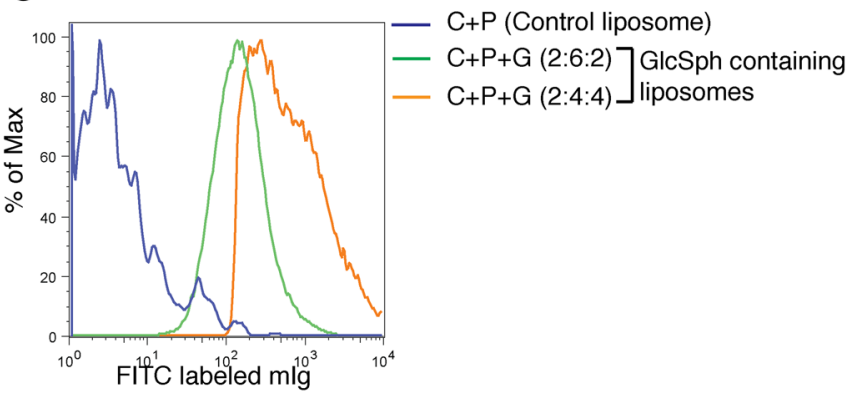

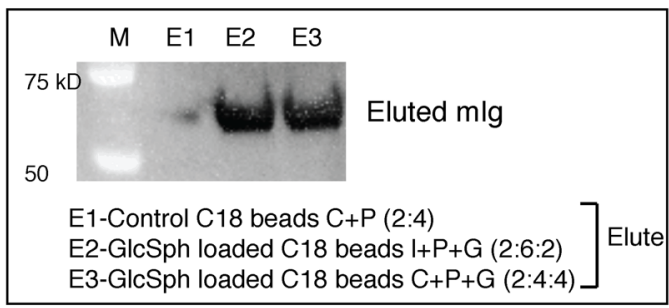

$\mathbf{F}$
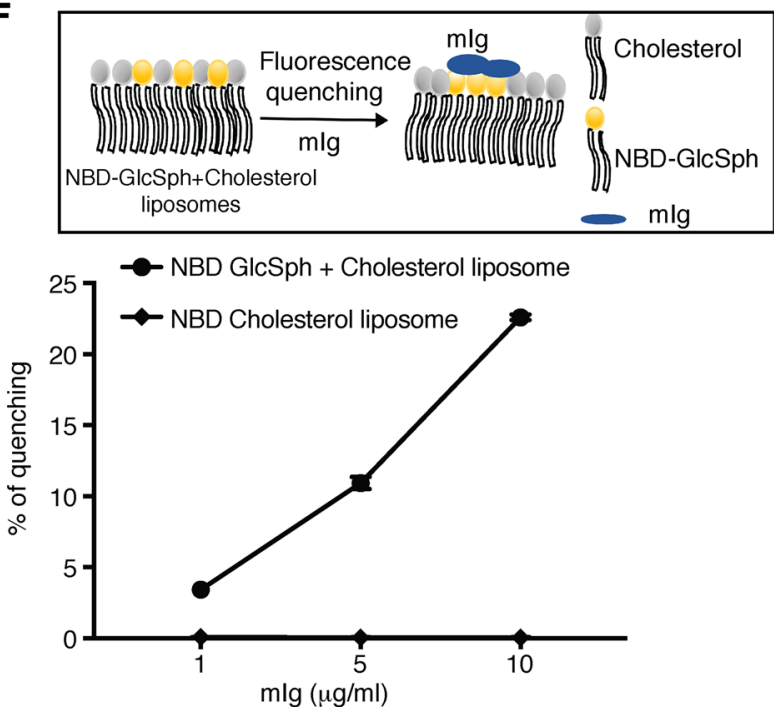

Figure 2. Binding of purified monoclonal Ig from lipid-reactive patients to GlcSph containing liposomes and C18 silica beads. (A) In the liposome sedimentation assay, purified monoclonal lg (mlgs) from lipid-reactive sporadic MM patient were incubated with either control or GlcSph containing liposomes and, after centrifugation, separated into liposome-bound fraction (B; 100\%) and liposome unbound fraction (S; 7.5\%) and visualized using mlg-specific anti-human heavy chain antibody in immunoblots. (B) Binding of mlgs from lipid-reactive GD patient to GlcSph containing liposomes as determined by liposome sedimentation assay. (C) Lipid binding specificity of mlg was assessed in a liposome-binding assay using flow cytometry. Representative FACS profile shows the dose-dependent binding of FITC-labeled affinity purified mlg to GlcSph containing liposomes. Data from 1 representative patient is shown. (D) Binding of the FITC-labeled mlg from sporadic MM patient to GlcSph-coated C18 silica beads was assessed by flow cytometry (left). Bound clonal Ig was eluted using $1 \mathrm{M}$ glycine ( $\mathrm{pH} 2.0_{-}$, the beads were checked after elution by FACS, while the eluted fraction was visualized by immunoblotting (right). (E) Clonal Ig from lipid-reactive GD patient also show binding to GlcSph-coated C18 silica beads. (F) Percent quenching of the relative fluorescence intensity of NBD-GlcSph in NBD-GlcSph/cholesterol liposomes induced by GlcSph binding of $\mathrm{mlg}$ from lipid-reactive patient. No fluorescence quenching of NBD-cholesterol control liposome is observed in the presence of mlg. Data represent mean \pm SEM. Data is representative of 4 similar experiments.

flow cytometry. We observed concentration-dependent binding of clonal Ig to GlcSph-coated beads as compared with control beads (Figure 2D). To test if fluorescence signal associated with GlcSph-containing C18 beads was due to mIgs, GlcSph -bound Igs were eluted using $1 \mathrm{M}$ glycine buffer ( $\mathrm{pH}$ 2.0). Immunoblots probed with specific anti-heavy chain antibody showed the presence of Igs in the eluted fraction, while corresponding 
ASTKCPSVFP LAPSSKSTSC GTAALCCLVK DYFPEPVTVS WNSGALTSCV HTFPAVLQSS GLYSLSSVVT VPSSSLCTQT YICNVNHKPS NTKVDKKVEP KSCDKTHTCP PCPAPELLCC
PSVF

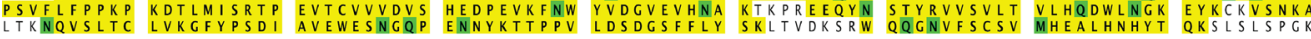

Liposome bound $\lg (88 \%$ coverage)

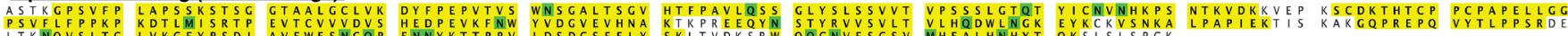

Sphingosine beads bound sera $(60 \%$ coverage)

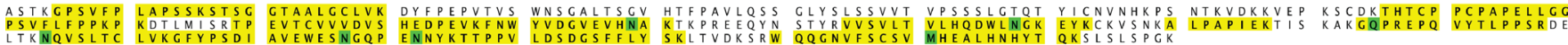

Lipid reactive $\mathrm{R} 2(\lg A)$

mlg $(85 \%$ coverage)

Human Ig alpha-1 protein sequence

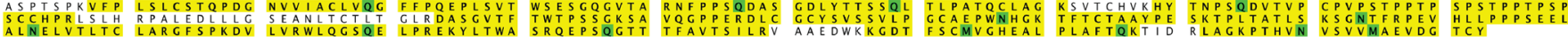

Liposome bound $\lg (90 \%$ coverage)

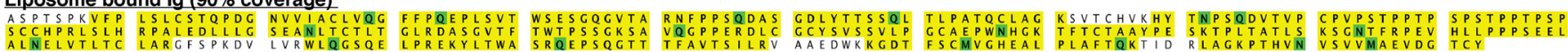

Sphingosine beads bound sera (95\% coverage)

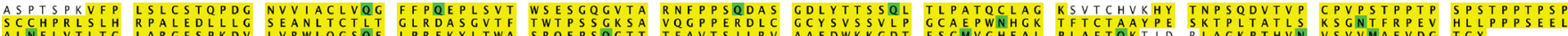

The sequences in yellow show the matched peptides

B

Lipid reactive $\mathrm{R} 2$

Heavy chain c-DNA translated sequence

mlg

DNAKNALYLQLNSLRVEDTAVYYCARDLCYYGSGRCYNDDAFDIWGQGT

Liposome bound Ig

DNAKNALYLQLNSLRVEDTAVYYCARDLCYYGSGRCYNDDAFDIWGQGT

Sphingosine beads bound sera

DNAKNALYLQLNSLRVEDTAVYYCARDLCYYGSGRCYNDDAFDIWGQGT

TLTISSLQSEDFAVYYCQQYNIWPPITFGQGTR

Light chain C-DNA translated sequence

CDR3

TLTISSLQSEDFAVYYCQQYNIWPPITFGQGTR

TLTISSLQSEDFAVYYCQQYNIWPPITFGQGTR

The sequences in yellow show the matched peptides

Figure 3. Protein sequence analysis by mass spectrometry between purified clonal Ig and Ig eluted from GlcSph-containing liposomes. (A) Protein sequences obtained after LC/MS from purified monoclonal $\mathrm{lg}(\mathrm{mlg})$, liposome-bound protein, and protein eluted from sphingosine beads from 2 different lipid-reactive patients are shown. (B) Protein sequences obtained after LC/MS from purified clonal Ig, liposome-bound protein, and protein eluted from sphingosine beads from R2 match with the heavy and light chain CDR3 region of the cDNA translated sequence obtained from the rlg cloned from single plasma cells of the same patient. LC/MS protein sequencing was performed on 3 patients' samples.

fluorescence signal associated with GlcSph-containing C18 beads was lost (Figure 2D). Purified Igs from a GD patient also showed binding to GlcSph-loaded C18 silica beads (Figure 2E). The above observations suggest that purified Igs from both sporadic MM and GD patients with LRG preferentially bound to both GlcSph-containing liposomes and C18 silica beads. To further substantiate that clonal Ig interacts with GlcSph-containing liposomes, fluorescence quenching assay was performed using nitrobenzoxadiazole-labeled (NBD-labeled) GlcSph and cholesterol liposome with NBD-labeled cholesterol liposomes as control. A dose-dependent quenching of NBD-labeled GlcSph fluorescence but not of NBD-labeled cholesterol fluorescence indicated specific binding of clonal Ig with GlcSph (Figure 2F). Together, these data obtained using several complementary approaches for testing Ig/lipid interactions provide direct evidence that Igs from LRG patients belonging to both sporadic MM and GD have the capacity to bind lysolipids incorporated in a physiologically relevant (membrane-integrated) format.

Protein sequencing and identification by MS of purified clonal Igs and eluted Ig from GlcSph-containing liposomes. To unambiguously establish the clonal nature of Igs bound to GlcSph liposomes or sphingosine beads, we analyzed the protein sequence of mIg purified from patient sera and lysolipid binding Ig eluted from GlcSph liposomes or beads by MS. Analysis of tryptic digests by MS/MS sequencing demonstrated high sequence identity between the purified $\mathrm{mIg}$, Ig bound to GlcSph liposomes, and Ig eluted from sphingosine beads (Figure 3A). In line with these results, protein sequences from lysolipid-bound Igs also contained complementarity determining region 3 (CDR3) of rIg cloned from single 
A

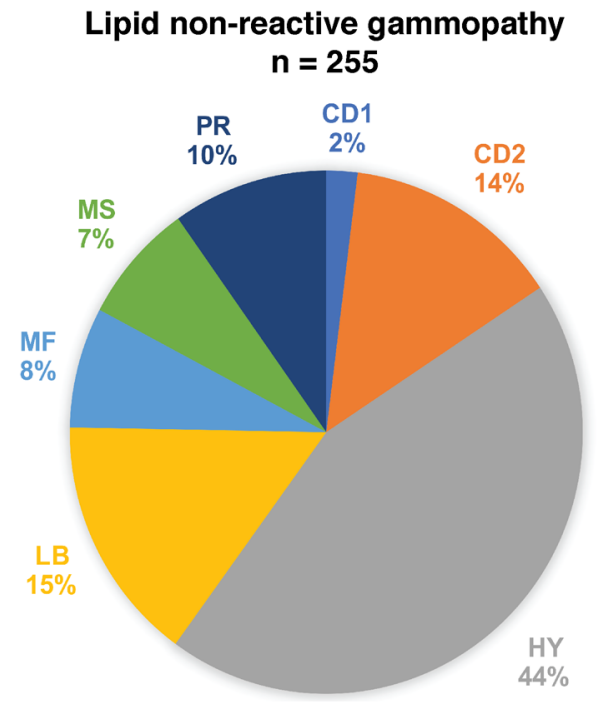

Lipid reactive gammopathy

$$
\mathrm{n}=95
$$

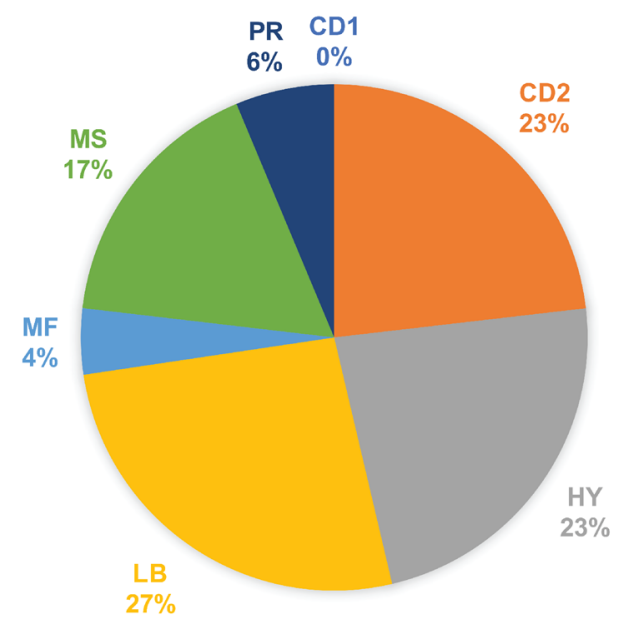

B

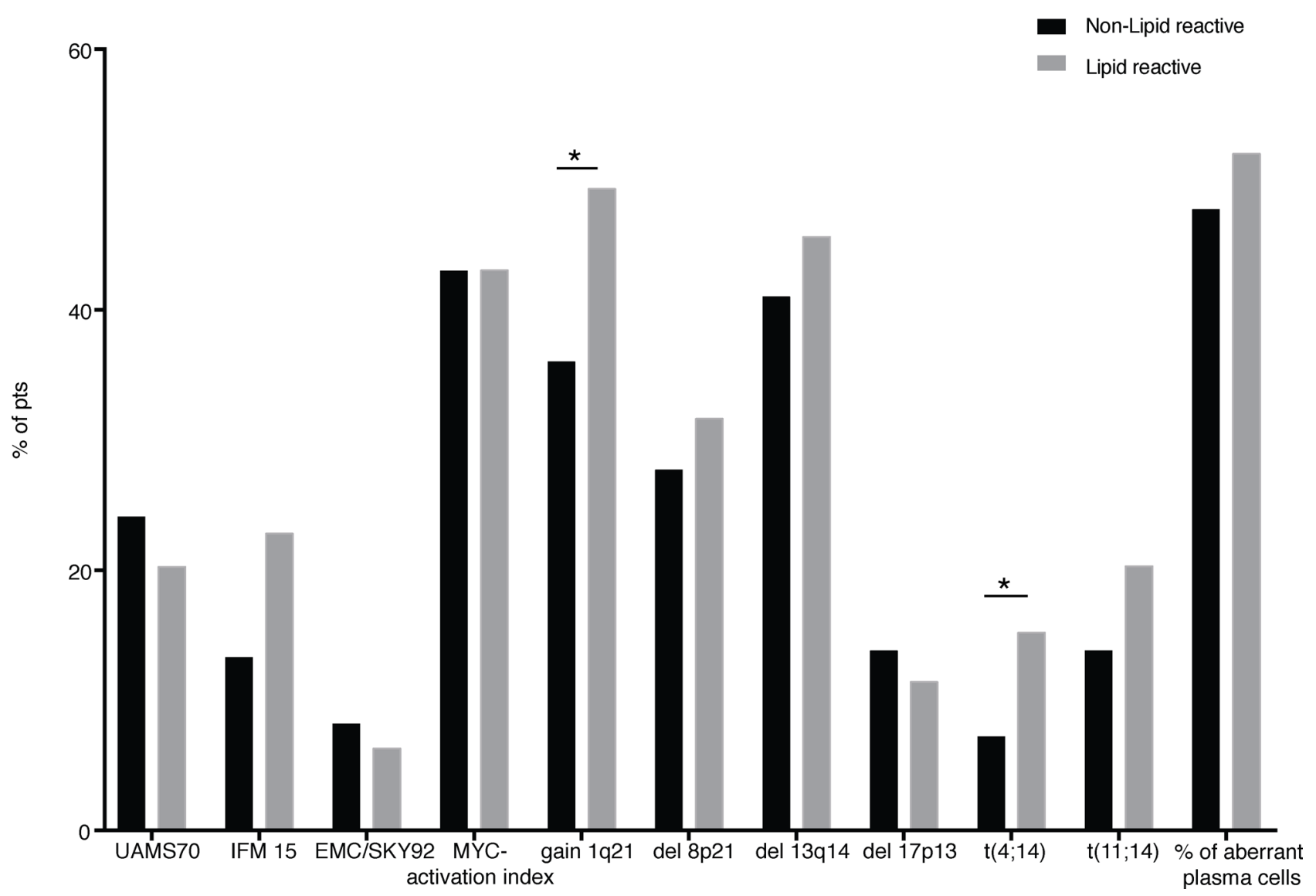

Figure 4. Genetic features of tumor cells in lipid-reactive gammopathy. (A) Pie charts compare the percentages of different molecular classification (MC) subgroups between lipid-reactive and non-lipid-reactive patients. Data shown are cumulative data from cohort $1(n=76)$ and $2(n=274)$ ${ }^{* * *} P<0.001,{ }^{*} P<0.05$, Fisher's exact test). (B) Bar graph shows comparison of percentage of patients based on the detection of high-risk GEP signatures and cytogenetic abnormalities in tumor cells between non-lipid-reactive and lipid-reactive patients. Data are from patients in cohort 2 $(n=274)$ with available cytogenetics and GEP signatures ( ${ }^{*} P<0.05$, Fisher's exact test). HY, hyperdiploid; LB, low bone; MF, Maf; MS, MMSET; PR, proliferation; CD, cyclin D. 
tumor cells tested in Figure 1A (Figure 3B). Together, these results unequivocally demonstrate that lysolipid-binding Igs are clonal in nature.

Lipid-reactive gammopathies have lower proportion of HY tumors. Our next goal was to see if the lipid-reactive group of patients was associated with any of the MM molecular subtypes. To this end, we analyzed the presence of lipid-reactive clonal Ig in plasma/sera from 2 independent cohorts of patients with gammopathy. Patients in cohort 1 with asymptomatic MM or MGUS were enrolled in SWOG trial S0120 (5), while those in cohort 2 (University of Heidelberg) received therapy for active MM. Overall, the presence of LRG was noted in 53 of $216(24.5 \%)$ of evaluable patients in cohort 1, and 79 of 274 (28\%) of evaluable patients in cohort 2. Data for GEP/genetics of tumor cells was available for 76 patients in cohort 1 and all patients in cohort 2. Both cohorts contained patients with the known 7 molecular subtypes of MM $(5,9)$ and were pooled to analyze the genetic correlates of LRG. Patients with LRG had a significantly lower proportion of patients with HY subset, indicating that LRG is particularly enriched within the non-HY subset, typically associated with IgH translocations (Figure 4A and Supplemental Table 2). In contrast, LRG was associated with a higher proportion of patients with cyclin D type 2 (CD-2), MMSET group, and low bone disease (LB) subsets. Consistent with the data on molecular subtypes, cytogenetic analyses revealed that a lower proportion of patients with LRG had HY tumors compared with the nonreactive cohort and that patients with LRG and a higher proportion of $\mathrm{t}(4: 14)$ and gain of 1q21 compared with the nonreactive cohort (Figure 4B and Supplemental Table 3). Proportion of patients with high risk gene expression signatures were comparable in the LRG and NRG subsets. Thus, GEP and cytogenetic analyses showed enrichment of non-HY tumors in lipid-reactive patients.

Reactivity of clonal plasma cell and Ig to lipid antigen in vivo. In prior studies, we have shown that MISTRG6 mice can serve as effective hosts for the growth of human MGUS and MM cells in vivo (17). We therefore utilized these mice to grow BM mononuclear cells (MNCs) from a patient with known lipid-reactive IgG $\kappa$ GD-MG in vivo. Growth of $\kappa$-restricted clonal PCs in the BM was associated with suppression of nonclonal $\lambda$-expressing PCs. Injection of GlcSph led an increase in clonal Ig (M spike) compared with PBS controls, as detected by serum protein electrophoresis (Figure 5A). Injection of GlcSph also led to an increase in $\kappa$-expressing PCs in vivo, as well as some increase in nonclonal $\lambda$-restricted PCs (Figure $5, \mathrm{~B}$ and C). Importantly, $\kappa$-restricted PCs had an aberrant phenotype with aberrant expression of CD33 and CD56, in contrast to nonclonal PCs, utilized in some studies to differentiate normal from abnormal plasma cells (Figure 5D) (18). Overall, these data demonstrate that antigenic stimulation led to an increase in clonal PCs and clonal Igs in vivo and also reactivated previously suppressed nonclonal PCs and Igs.

\section{Discussion}

Herein, our data provide direct evidence that lipid-reactive clonal Ig in patients with GD-MG and a subset of sporadic MM is derived from clonal plasma cells. These studies also establish the capacity of clonal Igs to bind lipid antigens presented in a physiologic context, incorporated into a membrane bilayer. We further demonstrate that plasma cells proliferate when stimulated with lysolipids in vivo. Together, these data provide definitive evidence that the cell of origin in these gammopathies is a lipid-reactive B or plasma cell.

The precise biochemical nature and source (exogenous or endogenous) of specific lysolipids that serve as antigenic targets of clonal PCs remains an area of active investigation. In prior studies, we have shown that the clonal Ig exhibits cross-reactivity with related lysolipids such as GlcSph and lyso-PC (LPC) (14, 19). GlcSph is markedly increased in GD patients, and persistent stimulation by the accumulated lipid could contribute to GD-associated gammopathy. Prior studies have demonstrated elevated levels of LPC and LPC-reactive T cells in some MM patients $(19,20)$. Elevated concentration of LPC in the sera of MM patients and its association with inflammation (21) make LPC a candidate as a potential antigen for LRG in some patients with sporadic (non-GD) MM.

$\mathrm{MM}$ is a heterogeneous malignancy with genetically distinct subtypes that differ in clinical presentation and outcome (2). However, whether the individual MM types have distinct etiopathogenetic origins is not known. The findings in this manuscript suggest that molecular subtypes of MM may differ in their etiopathogenesis with enrichment of LRG in non-HY tumors. Whether distinct subtypes of non-HY MM (such as CD-2 or t [4:14]) are particularly enriched in LRG requires validation in additional cohorts. It is notable that tumor cells in CD-2 subsets aberrantly express B cell markers (9), and the MMSET gene dysregulated in the MS subset carrying $\mathrm{t}(4: 14)$ has been implicated in class-switch recombination (22). It is also intriguing that LRG is associated with gain of 1q21, which correlates with higher proliferation index and 
A

A Tumor engrafted MIS $^{(K l)}$ TRG6mice
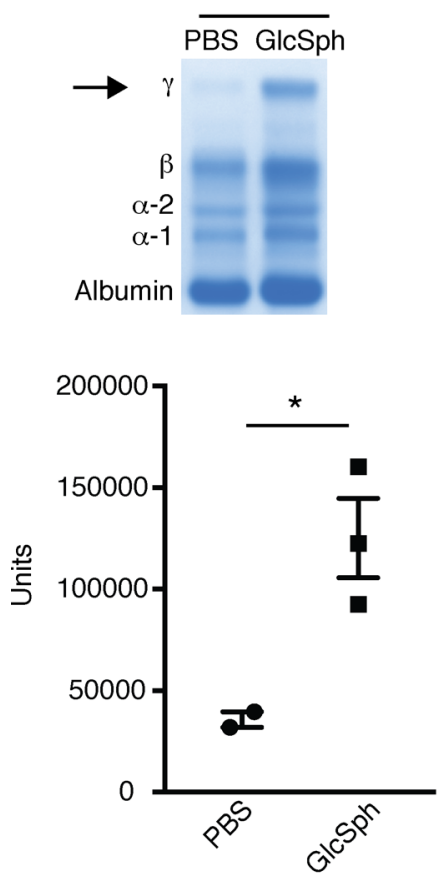

B
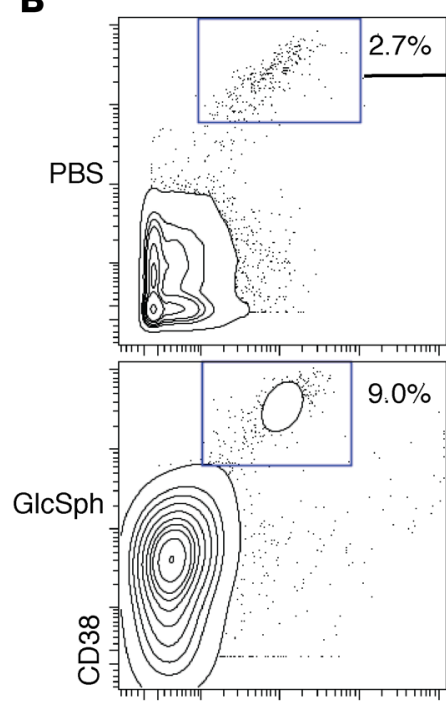

CD138

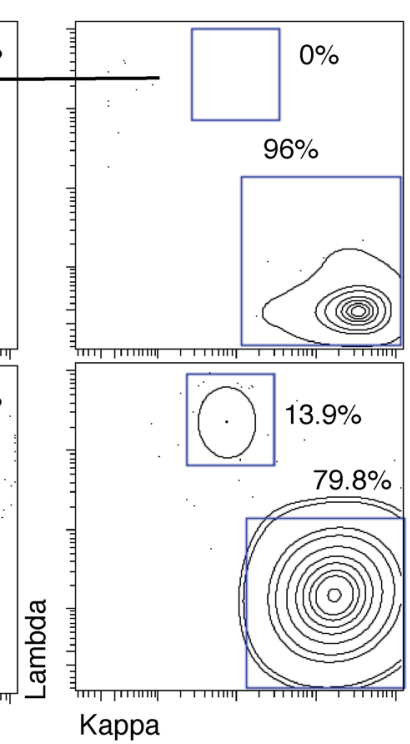

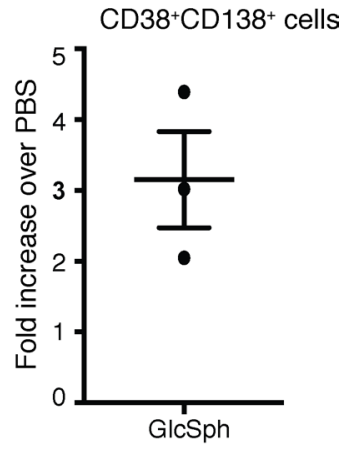

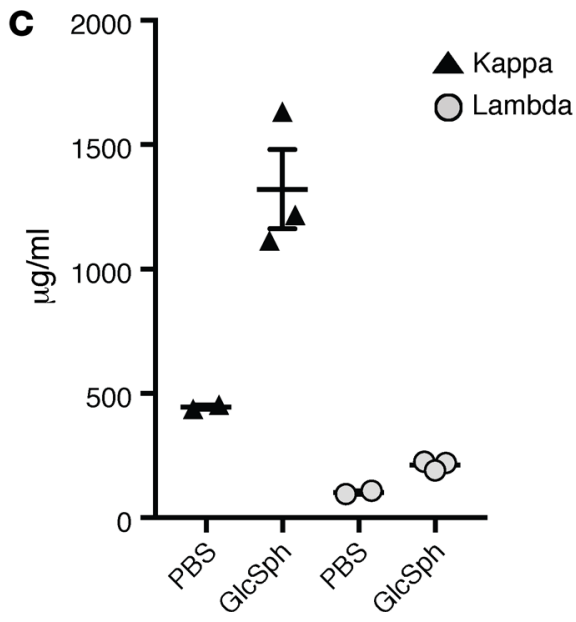

D

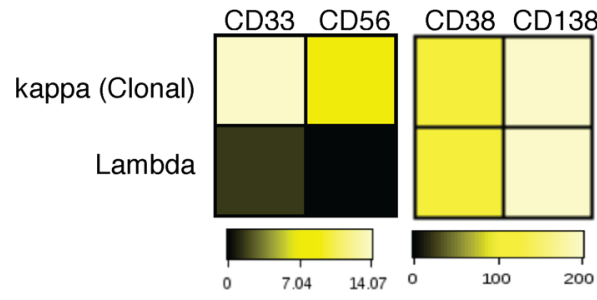

Figure 5. Reactivity of clonal plasma cells and Ig to lipid antigen in vivo. (A) Serum protein electrophoresis (SPEP) was performed on serum specimens obtained from tumor-engrafted MIS(K)TRG6 mice injected with either PBS or GlcSph. Fractions shown are $\alpha-1$ and -2 globulin, $\beta$ globulin, and $\gamma$ globulin. The arrow indicates the paraprotein (top panel). The bottom panel shows densitometry quantitation of paraprotein seen in PBS- vs. ClcSph-injected mice performed using Imagel software $(n=5)\left({ }^{*} P<0.05\right.$,Welch's $t$ test). (B) Representative contour plots show the percentage of human $\mathrm{CD} 38^{+}{ }^{-C D} 138^{+}$plasma cells and tumor light-chain profile among human cellular compartment (mCD45-mTer119-) in BM mononuclear cells obtained from tumor-engrafted (IgGK) mice after 3 weeks of injections with PBS or GlcSph (the gating strategy shown in Supplemental Figure 7). The dot plot shows the fold increase in CD38+CD138+ plasma cells in BM of engrafted mice after injection of GlcSph over those injected with PBS $(n=3)$. (C)The dot plot shows the levels of human light-chain-restricted antibodies $(\mu \mathrm{g} / \mathrm{ml})$ in mouse sera, detected by ELISA. Data represent mean \pm SEM $(n=3)$. (D) Heatmap for the expression of CD33, CD56, CD38, and CD138 on clonal $\kappa$ and nonclonal $\lambda$ plasma cells in GlcSph-injected tumor engrafted mice as in B, as analyzed by mass cytometry (CyTOF). Bars represent expression scales.

high-risk MM $(23,24)$. Amplification of this locus typifies high-risk MM and contains several candidate genes. Interestingly, this locus also harbors GBA (a gene defective in GD) and a closely related pseudogene that confers vulnerability to gene conversion events and transfer for disease-causing mutations in the active gene (25). Thus, the amplified 1q21 locus may lead to alteration in lipid homeostasis in tumor cells that may further drive myelomagenesis. Several mechanisms may underlie the induction of genomic instability and cellular transformation in the setting of lipid-mediated inflammation. This may include potential effects of lipids on the activation of endogenous retroviruses (26), effects on cellular membranes, DNA damage 

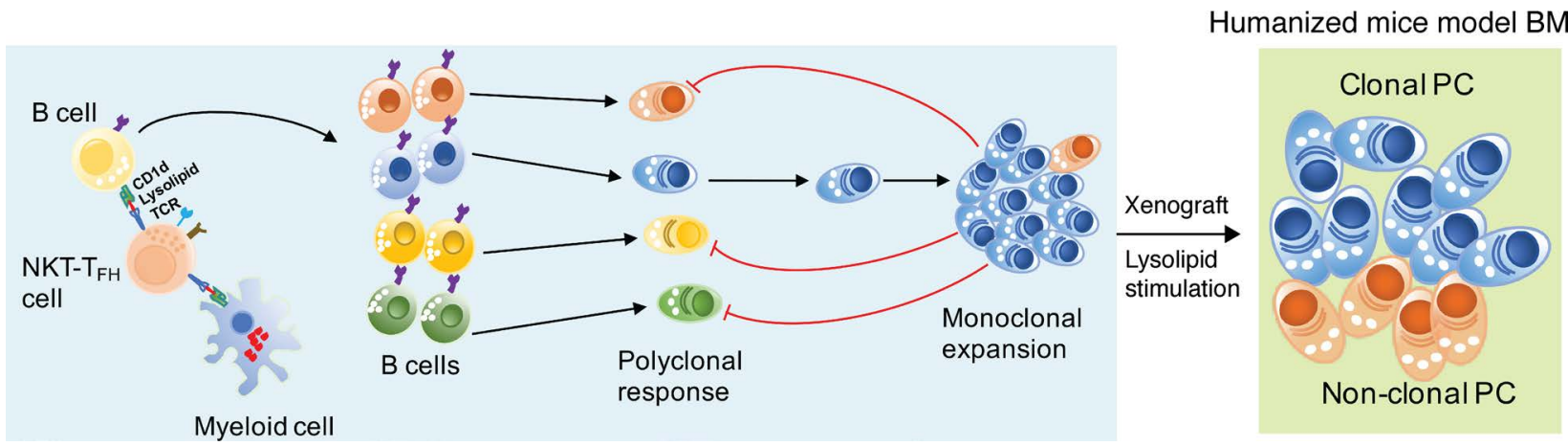

Figure 6. Proposed model to show lysolipid-mediated stimulation of clonal and nonclonal plasma cells. Proposed model depicting progression of GD gammopathy from initial lysolipid-reactive polyclonal response and emergence of monoclonal PCs through suppression of antigenically related nonclonal $\mathrm{PCs}$ in vivo. Antigenic restimulation with lysolipids was able to overcome this suppression, leading to activation of both clonal and nonclonal plasma cells in vivo in a humanized mouse model.

induced by inflammation-associated reactive oxygen/nitrogen species, or induction of cytidine deaminases such as AID, implicated in the generation of IgH translocations in murine models (27-29). Indeed, it may be the capacity of lipids to also mediate chronic inflammation that distinguishes them from chronic viral infections that do not commonly lead to plasma cell tumors. It should be noted that aberrant activation of cytidine deaminases and resultant genomic instability may lead to ongoing mutations in the Ig genes with disease progression $(28,30)$ and could, in principle, even lead to changes in antigen reactivity of clonal Ig or B cell receptors over time (31).

Overall, these data support a model wherein chronic immune activation via lipid antigens may lead to expansion and eventually transformation of lipid-reactive B/plasma cells in a subset of MM patients. An implication of this model is that monoclonality emerges due to acquisition of genetic/epigenetic changes in the founder clone in the setting of an initial oligoclonal/polyclonal response to a specific antigen. This is recapitulated in GD gammopathy, wherein the underlying polyclonal gammopathy also has lipid-reactivity. Notably, our data in a humanized mouse model engrafted with GD-MG tumor cells show that antigen stimulation can activate both clonal and nonclonal plasma cells. Therefore, these data support a model wherein GD-associated monoclonal gammopathy originates as part of a polyclonal response against antigenic lipids, and emergence of monoclonal PCs is associated with suppression of antigenically related nonclonal PCs. However, antigen stimulation can overcome this suppression and activate both clonal and nonclonal plasma cells (Figure 6). Further studies are needed to test if antigenically related polyclonal PCs also exist in lipid-reactive sporadic MM and respond to antigen-mediated stimulation in vivo. These data also provide proof of principle that the malignant clone may remain responsive to antigen-mediated stimulation in vivo. This finding has potential clinical implications for developing novel approaches targeting the underlying antigen to prevent the progression or evolution of gammopathy. Newly approved therapies can lead to pharmacologic reduction in lysolipid-antigen substrates in GD patients (32) and should be evaluated for their impact on GD gammopathy.

\section{Methods}

Patients. Patients with IgG/IgA MGUS or asymptomatic MM were included in an observational clinical trial SWOG (trial S0120) and constituted cohort 1 (5). Cohort 2 consisted of patients with active myeloma (University of Heidelberg). Total RNA from these purified CD $138^{+}$PCs was used to measure GEP with Affymetrix U133 Plus 2.0 microarrays. Cytogenetic and FISH analysis data were available for patients in cohort 2.

Lipids. Egg L-a-PC, and ovine wool cholesterol, all in chloroform, were purchased from Avanti Polar Lipids Inc. GlcSph and NBD-GlcSph were purchased from Matreya, while NBD cholesterol was purchased from Thermo Fisher Scientific.

Purification of $\mathrm{IgG} / \mathrm{A}$ from patient sera. For some of the sera sample analyzed in this study, $10 \mathrm{ml}$ of diluted sera (1:2 dilution in PBS; MilliporeSigma) was passed through a 4-ml Protein A/G Ultralink resin (Thermo Fisher Scientific) affinity column in gravity mode. Plasma flow through was collected and passed through the column 3 times. The column was washed with 15 column volumes (cv) of PBS before elution 
with $5 \mathrm{cv}$ of $100 \mathrm{mM}$ glycine- $\mathrm{HCl}(\mathrm{pH}$ 2.7). The eluate, containing total IgG/A from plasma, was immediately neutralized with $2 \mathrm{ml}$ of $1 \mathrm{M}$ Tris- $\mathrm{HCl}(\mathrm{pH} 8.0)$ and dialyzed into $20 \mathrm{mM}$ sodium acetate ( $\mathrm{pH} 4.5)$ overnight at $4^{\circ} \mathrm{C}$. The purity of the purified IgG/A was checked by native PAGE, and the band corresponding to the Ig molecular weight was cut and given for LC/MS analysis. Heavy and light chain specificity of the purified Ig with the corresponding patient sera was verified using IEF and immunoblotting. Further, the lipid-binding capacity of the purified Ig with the corresponding patient plasma was checked using GlcSph-specific immunoblotting.

Generation of $m$ Abs from plasmablasts. Recombinant $\mathrm{mAbs}$ from single patient plasma cells was generated according to the protocol described earlier $(33,34)$. A schematic overview of the protocol has been outlined (Supplemental Figure 1). Briefly, CD138 cells from MNCs isolated from patient BM were enriched using anti-CD138 magnetic beads (Miltenyi Biotec) and stained with fluorescently labeled anti-human antibodies against CD138 (clone MI15), CD38 (clone HIT2), CD45 (clone HI130), CD19 (clone SJ25-C1), $\lambda$ (clone 1-155-2) (BD Pharmingen), and $\kappa$ (clone MHK-49) (BioLegend) to stain plasma cell populations. Single cells were sorted on FACSAria, excluding cell duplets and dead cells (Live/Dead Fixable Violet Dead Cell stain kit, Invitrogen, L34955) into 96-well PCR plates and immediately frozen on dry ice before storage at $-80^{\circ} \mathrm{C}$. cDNA from single cells was synthesized by reverse transcription PCR (RT-PCR) using random primers. Amplification of full-length $\operatorname{IgH}$ and $\operatorname{Ig} \kappa$ or $\operatorname{Ig} \lambda \mathrm{L}$ chain variable regions was achieved by using nested PCR. All the PCR products were then purified and sequenced. Clonally related sequences having identical germline V (D)J gene segments, and CDR3 sequence of $\operatorname{IgH}$ and $\operatorname{IgL}$ chain analyzed using the IgBlast or IMGT website (www.imgt.org) (Supplemental Table 1) were then cloned into eukaryotic expression vectors containing the human $\operatorname{Ig} \gamma 1$, Ig 1 , or $\operatorname{Ig} \lambda 2$ constant regions. The mIg were purified using affinity chromatography with Sepharose $G$ beads. For some experiments, $F(a b)_{2}$ fragment was purified by using Pierce F(ab')2 Preparation Kit (Thermo Fisher Scientific).

ELISA. GlcSph was diluted in ethanol $(2 \mathrm{mg} / \mathrm{ml})$ and $100 \mu \mathrm{l}$ (containing a total of $200 \mu \mathrm{g}$ ) was placed in each well of a Costar 96-well sterile round-bottom tissue culture plate. The plates were incubated overnight to allow ethanol to evaporate. PBS-boiled casein (0.5\%), pH 7.4, was added to each well as a blocking agent. rIg isolated from single-cell sorted purified patient plasma cells were serially diluted in blocking buffer and added to the plates overnight at room temperature. Plates were then washed and detected using perodixase-linked goat anti-human IgG (1:1,000) (Jackson ImmunoResearch; 109-035-008), since eukaryotic expression vector only contains human Ig 1 constant region. For competition ELISA, GlcSph-coated wells were incubated with different concentrations of recombinant B cell receptor (BCR) F(ab) from both LRG and non-LRG patients, followed by addition of lipid-reactive purified mIg from patient sera. Plates were then washed and detected using perodixase-linked anti-human IgH antibody $(1: 1,000)$ (AffiniPure goat anti-human serum IgA, $\alpha$ chain specific HRP conjugated [catalog 109-035-011]; AffiniPure goat antihuman serum IgG, HRP conjugated [catalog 109-035-003]; Jackson ImmunoResearch).

Liposomes. Liposomes constituted with only GlcSph were found to form aggregates and were unstable when checked by DLS. The likely reason for the observed instability of the GlcSph liposome could be the single carbon chain nature of GlcSph. Therefore, we incorporated cholesterol and PC with GlcSph to make unilamellar liposomes. As a control, liposomes containing cholesterol and PC without GlcSph were utilized. Briefly, the lipids PC and cholesterol, with or without GlcSph (2 mg of total lipid), were diluted in chloroform and pipetted into a glass tube in one of the following ratios (w:w): cholesterol/PC (2: 4) for control liposomes or cholesterol/PC/GlcSph (2:6:2 or 2:4:4) for GlcSph-containing liposomes. Lipid mixtures were placed under a steady flow of nitrogen and then under an ultra-high vacuum for 1 hour to evaporate chloroform. Lipids were resuspended in $1 \mathrm{ml} \mathrm{PBS}$ to give a final lipid concentration of $2 \mathrm{mg} / \mathrm{ml}$. This solution was placed in a $65^{\circ} \mathrm{C}$ water bath for 5 minutes before being alternately placed into a dry ice-ethanol bath or $65^{\circ} \mathrm{C}$ water bath for 3 minutes each. After 10 freeze-thaw cycles with intermittent vortexing, the resulting lipid solutions were passed 21 times through a $0.1-\mathrm{mm}$ polycarbonate filter using a Mini-Extruder from Avanti Polar Lipids Inc. The size and shape of the resulting particles were immediately characterized by DLS and were used in liposome-binding assay the same day. Concentration of GlcSph in the liposomes was estimated by Liquid chromatography-tandem MS (LC/MS/MS) (Supplemental Figure 6).

Liposome-binding assay. The interaction between purified paraprotein and liposome was analyzed by liposome sedimentation assay. Purified paraprotein $(20 \mu \mathrm{g})$ was added to either control or GlcSph-containing liposomes and incubated for 30 minutes at room temperature. Liposomes were harvested by centrifugation at 50,000 $g$ for 20 minutes in Beckman Coulter Optima Ultracentrifuge with TLA120.2 rotor. After supernatant 
was carefully removed, the pellet was washed with $500 \mu \mathrm{l}$ of PBS, and the pelleted protein was bound to liposome (bound fraction, 100\%). Protein in the supernatant (unbound fraction, $7.5 \%$ ) was analyzed by SDS-PAGE, followed by Western blot using anti-human heavy chain specific HRP antibodies (FITC mouse anti-human IgG [clone G18-145, BD Biosciences]; goat anti-human IgA allophycocyanin [catalog ab99897; Abcam]), using modified protocol from previous work (35). For some samples, the sedimented liposome protein was resuspended in PBS and given for LC/MS analysis. In some experiments, liposome-bound paraprotein was detected by FACS using either FITC-labeled paraprotein or by fluorochrome-conjugated anti-IgH secondary Ab. Flow cytometry was accomplished using a BD FACSCalibur Flow Cytometer. Twenty thousand events were acquired per sample and analyzed using CELLQUEST software.

Sphingosine bead-protein pull down. Human plasma at a 1:50 dilution was incubated with $400 \mu 1$ of sphingosine-coated beads (Echelon Biosciences) on a shaker overnight at $4^{\circ} \mathrm{C}$. The beads were washed 4 times with the co-IP buffer (10 mM HEPES [Thermo Fisher Scientific], pH 7.4; 150mM NaCl [Thermo Fisher Scientific]; $0.25 \%$ IGEPAL [MilliporeSigma]), and the bound proteins were eluted using $400 \mu 1$ of $100 \mathrm{mM}$ glycine-HCL, pH2.7. The eluted fraction was then analyzed by LC/MS.

Lipid-coated C18 bead preparation. C18 silica beads are octadecyl modified silica beads with nonpolar, endcapped phase with hydrophobic C18 Alkyl groups on their surface. C18 silica beads (2 mg; MilliporeSigma; pore size $100 \AA$; particle size 15-25 $\mu \mathrm{m}$ ) and YMC America Inc. (pore size, $12 \mathrm{~nm}$; particle size, $5 \mu \mathrm{m}$ ) were measured and suspended in $100 \mu 1$ of chloroform. Control C18 beads were loaded with cholesterol and PC at a 2:4 ratio, while GlcSph-containing C18 beads were mixed with cholesterol/PC/GlcSph at 2:6:2 or 2:4:4 ratios to make total lipid concentration $2 \mathrm{mg} / \mathrm{ml}$. After mixing, the lipid-C18 bead mixture was dried under nitrogen. The bead lipid mixture was then rehydrated with $20 \mathrm{mM}$ Tris- $\mathrm{HCl}$ (Thermo Fisher Scientific), vortexed, sonicated until dispersed, and washed several times before use as described previously by Yeung et al. (36). GlcSph concentration bound to the $\mathrm{C} 18$ beads (approximately $500 \mu \mathrm{g} / \mathrm{ml}$ of GlcSph) was estimated by using LC/MS/MS (Supplemental Figure 6). Lipid-coated beads were incubated with either FITC-conjugated purified Ig or unlabeled Ig (1:150) for 30 minutes at $37^{\circ} \mathrm{C}$. The bound antibody in case of unlabeled Ig was detected by using fluorochrome-conjugated anti-IgH secondary Ab (FITC mouse anti-human IgG [clone G18-145, BD Biosciences]; goat anti-human IgA allophycocyanin [catalog ab99897; Abcam]). Bead-associated fluorescence was measured with flow cytometry. Concurrently, lipid-coated bead bound purified Ig was eluted and Western blot was run to confirm the heavy chain specificity of the bound purified Ig.

NBD quenching. Relative fluorescence of NBD-cholesterol (control liposome) and cholesterol/ NBD-GlcSph (1:1) liposomes was measured by excitation at $465 \mathrm{~nm}$ and emission at $530 \mathrm{~nm}$ using Spectramax M3 spectrofluorometer. All measurements were performed at $25^{\circ} \mathrm{C}$. For each measurement, $200 \mu 1$ of liposomes was used and desired amounts of protein were titrated into the liposomes and incubated for 10 minutes before each measurement. Quenching percentage was calculated by the equation (Fo $-\mathrm{F}) / \mathrm{Fo}$ $\times 100$, where $\mathrm{F}$ and Fo are fluorescence intensity in the presence and absence of protein, respectively (37). The fluorescence fluctuations due to the change in volume produced by the addition of protein solution were corrected by subtracting the value obtained by adding the same volume of buffer without protein.

Injection of patient BM cells into MIS ${ }^{(K I)}$ TRG6 mice. Generation of human M-CSF, IL-3, GM-CSF, thrombopoietin, and SIRP $\alpha$-knock-in mice (MIS ${ }^{[\mathrm{KI}]} \mathrm{TRG} 6$ mice) has been described earlier (17). BM samples were obtained from patients with GD with monoclonal gammopathy. BM MNCs were purified from patient BM by Ficoll density gradient centrifugation. Recipient mice (8-week-old females) were engrafted with BM MNCs $(100,000)$ following previously described methods $(17)$. The mice were bled after 4 weeks, and the engraftment of primary tumor cells was determined based on the detection of human Ig. Human Ig $\lambda$ and $\kappa$ levels were monitored using ELISA (Bethyl Laboratories Inc.), following the manufacturer's protocol. After 4 weeks of engraftment, the mice were given an i.p. injection of either PBS or GlcSph dissolved in PBS $(200 \mu \mathrm{g} / \mathrm{ml}$ in $100 \mu \mathrm{l})$. Three weeks' after lipid injection, the mice were bled, and paraprotein and human Ig levels in mice sera were analyzed by SPEP and ELISA, respectively, following manufacturer's protocol. The results presented are from an experiment, with a total of 3 mice/experimental group.

Immunophenotyping by mass cytometry. MNCs from the BM of the mice engrafted with patient BM MNCs were isolated following protocol described earlier. BM MNCs (up to 3 million/ $\mathrm{ml}$ ) isolated from the engrafted mice were stained for viability with Cell-ID Cisplatin ( $5 \mu \mathrm{M}$, DVS Sciences) for 1 minute at room temperature. Cells were then washed and stained with antibodies purchased either from Fluidigm or purchased purified and conjugated in-house using MaxPar X8 Antibody Labeling kit (Fluidigm) according to the manufacturer's instructions. A panel of anti-human antibodies (CD45-89Y, B7H3-141Pr, CD19-142Nd, 
HLADR-143Nd, CD69-144Nd, CD4-145Nd, CD8-146Nd, CD11c-147Sm, CD14-148Nd, CD200-149Sm, CD34-152Sm, TGIT-153Eu, CD27-155Gd, CXCR5-156Gd, CD33-158Gd, CCR7-159Tb, BTLA-163Dy, CD95-164Dy, CD45RO-165Ho, c-Kit-166Er, CD1b-167Er, CD25-169Tm, CD3-170Er, CD38-172Yb, CD138-173Yb, PDL1-174Yb, CD56-176Ybm and CD16-209Bi) was used along with anti-mouse CD45175Lu and Ter119-154Sm for surface staining (Supplemental Table 4). After incubation for 30 minutes at room temperature, cells were washed, fixed, and permeabilized using the $\mathrm{BD}$ Pharmingen Transcription Factor Buffer Set at $4^{\circ} \mathrm{C}$ overnight before being stained with intracellular antibodies (anti- $\lambda-151 \mathrm{Eu}$, anti- $\kappa$ 160Gdy, anti-CTLA-4-161Dy, anti-FoxP3-162Dy, anti-Ki-67-168Er, and anti-Granzyme-171Yb; Supplemental Table 4) for 30 minutes at room temperature. After intracellular staining, cells were washed and incubated in $0.125 \mathrm{~nm}$ Ir intercalator (Fluidigm) diluted in MaxPar Fix and Perm buffer and stored at $4^{\circ} \mathrm{C}$ until acquisition. Data was acquired on a CyTOF 2 instrument (DVS, Fluidigm Sciences Inc.) by detecting the signal from antibody-conjugated metal ions. After acquisition, the data were normalized using beadbased normalization in the CyTOF software. DVS Cytobank software (Cytobank Inc.) was used to analyze mass cytometry data. The gating was done to exclude any dead cells and doublets, as well as mouse cells, leaving DNA ${ }^{+} \mathrm{CD}_{4} 5^{+}$Cisplation $^{\text {lo }}$ events for subsequent analysis (Supplemental Figure 7).

Sample preparation for $L C / M S / M S$. The samples were subjected to methanol-chloroform precipitation. The precipitated protein pellets were resuspended in $8 \mathrm{M}$ Urea buffer $(10 \mu \mathrm{l})$, reduced by adding $2 \mu 1$ of 45 $\mathrm{mM}$ DTT and incubating at $37^{\circ} \mathrm{C}$ for 20 minutes, and they were alkylated by adding $2 \mu 1$ of $100 \mathrm{mM}$ iodoacetamide (IAA) and incubating at room temperature for 20 minutes. The samples were then diluted and digested with trypsin (Promega) for 16 hours at $37^{\circ} \mathrm{C}$. After acidifying to stop the digests, they were desalted by reversed phase chromatography using C18 MacroSpin columns (The Nest Group Inc.). C18 eluate was dried under vacuum centrifugation and resuspended in $50 \mu 1$ in $5 \%$ acetonitrile and $0.1 \%$ formic acid for MS analysis. In-gel digestion was performed according to the method reported by Shevchenko et al. (38).

Proteomics data analysis. All MS/MS samples were analyzed using the Sequest search engine embedded in the Proteome Discoverer software (version 2.1, Thermo Fisher Scientific). Sequest was set up to search SwissProt the human database (v2015-09-16; 20,193 entries), or cDNA sequence was translated to protein sequence. During the search, trypsin was specified as the digestion enzyme trypsin, with up to 2 allowed missed cleavage sites, fragment ion mass tolerance of $0.020 \mathrm{Da}$, and a parent ion tolerance of $10.0 \mathrm{PPM}$. Carbamidomethyl of cysteine was specified as a fixed modification, and deamidation of asparagine and oxidation of methionine were specified as variable modifications. Scaffold (version Scaffold_4.6.1, Proteome Software Inc.) was used to validate MS/MS-based peptide and protein identifications. Peptide identifications were accepted if they could be established at greater than $95.0 \%$ probability by the Peptide Prophet algorithm (39) with Scaffold $\Delta$-mass correction. Protein identifications were accepted if they could be established at greater than $99.0 \%$ probability and contained at least 2 identified peptides. Protein probabilities were assigned by the Protein Prophet algorithm (40). Proteins that contained similar peptides and could not be differentiated based on MS/MS analysis alone were grouped to satisfy the principles of parsimony.

Statistics. Data between lipid-reactive and non-lipid-reactive patients were compared using $\chi^{2}$ and Fisher's exact tests. Quantitative data is expressed as mean \pm SEM. A $t$ test was utilized as a 2-tailed test, and significance was set at $P<0.05$.

Study approval. All patient samples were collected after patients signed informed consent in accordance with protocols reviewed by the IRB of Yale University and the Declaration of Helsinki. All experiments were performed in compliance with Yale University Human Investigation Committee protocol and Yale IACUC protocols.

\section{Author contributions}

SN designed and performed experiments, analyzed data, and wrote the manuscript. JS, CSB, and LZ performed experiments and analyzed data. MC, MF, and ML performed some experiments. NR performed and analyzed MS data. AS and DH assisted with obtaining clinical specimens and analyzed data. NCM, RS, BB, RO, LB, TP, and NN performed clinical research. EM, PKM, and RAF designed research and analyzed data. MVD designed and directed overall research, analyzed data, and wrote the manuscript.

\section{Acknowledgments}

The authors thank Christopher Morgan and Xiaokui Zhang from Sanofi Genzyme for help with LC/MS/ MS analysis of liposomes and lipid-coated C18 beads, as well as Kavita Dhodapkar for discussions. We 
thank L. Devine and C. Wang for cell sorting. This work was supported in part by funds from the NIH R35 CA197603, Leukemia and Lymphoma Society Translational Research Program, and Multiple Myeloma Research Foundation-Perelman Family Foundation (all to MVD). PKM is supported in part by a Yale Center for Excellence in Clinical Translational Research in Gaucher Disease grant from Sanofi Genzyme.

Address correspondence to: Madhav V. Dhodapkar, HSRB E330 Emory University, 1760 Haygood Drive, Atlanta, Georgia 30322, USA. Phone:404.727.8372; Email: madhav.v.dhodapkar@emory.edu.

MVD's present address is: Winship Cancer Institute, Emory University, Atlanta, Georgia, USA.

1. Dhodapkar MV. MGUS to myeloma: a mysterious gammopathy of underexplored significance. Blood. 2016;128 (23):2599-2606.

2. Palumbo A, Anderson K. Multiple myeloma. N Engl J Med. 2011;364(11):1046-1060.

3. Walker BA, et al. Intraclonal heterogeneity is a critical early event in the development of myeloma and precedes the development of clinical symptoms. Leukemia. 2014;28 (2):384-390.

4. López-Corral L, et al. The progression from MGUS to smoldering myeloma and eventually to multiple myeloma involves a clonal expansion of genetically abnormal plasma cells. Clin Cancer Res. 2011;17 (7):1692-1700.

5. Dhodapkar MV, et al. Clinical, genomic, and imaging predictors of myeloma progression from asymptomatic monoclonal gammopathies (SWOG S0120). Blood. 2014;123 (1):78-85.

6. Zhao S, et al. Serial exome analysis of disease progression in premalignant gammopathies. Leukemia. 2014;28 (7):1548-1552.

7. Fonseca R, et al. Genetics and cytogenetics of multiple myeloma: a workshop report. Cancer Res. 2004;64 (4):1546-1558.

8. Fonseca R, et al. The recurrent IgH translocations are highly associated with nonhyperdiploid variant multiple myeloma. Blood. 2003;102 (7):2562-2567.

9. Zhan F, et al. The molecular classification of multiple myeloma. Blood. 2006;108(6):2020-2028.

10. Anderson PN, Potter M. Induction of plasma cell tumours in BALB-c mice with 2,6,10,14-tetramethylpentadecane (pristane). Nature. 1969;222(5197):994-995.

11. Mistry PK, Taddei T, vom Dahl S, Rosenbloom BE. Gaucher disease and malignancy: a model for cancer pathogenesis in an inborn error of metabolism. Crit Rev Oncog. 2013;18 (3):235-246.

12. Taddei TH, et al. The underrecognized progressive nature of N370S Gaucher disease and assessment of cancer risk in 403 patients. Am J Hematol. 2009;84(4):208-214.

13. Nair S, et al. Type II NKT-TFH cells against Gaucher lipids regulate B-cell immunity and inflammation. Blood. 2015;125 (8):1256-1271.

14. Nair S, Branagan AR, Liu J, Boddupalli CS, Mistry PK, Dhodapkar MV. Clonal Immunoglobulin against Lysolipids in the Origin of Myeloma. N Engl J Med. 2016;374 (6):555-561.

15. Pavlova EV, et al. Inhibition of UDP-glucosylceramide synthase in mice prevents Gaucher disease-associated B-cell malignancy. J Pathol. 2015;235 (1):113-124.

16. VanderVen BC, Hermetter A, Huang A, Maxfield FR, Russell DG, Yates RM. Development of a novel, cell-based chemical screen to identify inhibitors of intraphagosomal lipolysis in macrophages. Cytometry A. 2010;77 (8):751-760.

17. Das R, et al. Microenvironment-dependent growth of preneoplastic and malignant plasma cells in humanized mice. Nat Med. 2016;22 (11):1351-1357.

18. Kumar S, Kimlinger T, Morice W. Immunophenotyping in multiple myeloma and related plasma cell disorders. Best Pract Res Clin Haematol. 2010;23(3):433-451.

19. Chang DH, et al. Inflammation-associated lysophospholipids as ligands for CD1d-restricted T cells in human cancer. Blood. 2008;112(4):1308-1316.

20. Sasagawa T, Okita M, Murakami J, Kato T, Watanabe A. Abnormal serum lysophospholipids in multiple myeloma patients. Lipids. 1999;34 (1):17-21.

21. Salio M, Cerundolo V. Linking inflammation to natural killer T cell activation. PLoS Biol. 2009;7 (10):e1000226.

22. Pei H, Wu X, Liu T, Yu K, Jelinek DF, Lou Z. The histone methyltransferase MMSET regulates class switch recombination. J Immunol. 2013;190 (2):756-763.

23. Hose $\mathrm{D}$, et al. Proliferation is a central independent prognostic factor and target for personalized and risk-adapted treatment in multiple myeloma. Haematologica. 2011;96 (1):87-95.

24. Neben $\mathrm{K}$, et al. Progression in smoldering myeloma is independently determined by the chromosomal abnormalities del (17p), $\mathrm{t}$ (4;14), gain 1q, hyperdiploidy, and tumor load. J Clin Oncol. 2013;31 (34):4325-4332.

25. Horowitz M, Wilder S, Horowitz Z, Reiner O, Gelbart T, Beutler E. The human glucocerebrosidase gene and pseudogene: structure and evolution. Genomics. 1989;4 (1):87-96.

26. Knittel G, et al. Insertional hypermutation in mineral oil-induced plasmacytomas. Eur J Immunol. 2014;44 (9):2785-2801.

27. Robbiani DF, et al. AID is required for the chromosomal breaks in c-myc that lead to c-myc/IgH translocations. Cell. 2008;135 (6):1028-1038.

28. Cowan G, et al. Massive parallel IGHV gene sequencing reveals a germinal center pathway in origins of human multiple myeloma. Oncotarget. 2015;6 (15):13229-13240.

29. Colotta F, Allavena P, Sica A, Garlanda C, Mantovani A. Cancer-related inflammation, the seventh hallmark of cancer: links to genetic instability. Carcinogenesis. 2009;30 (7):1073-1081.

30. Munshi NC, et al. Deep IgH sequencing identifies an ongoing somatic hypermutation process with comples and evolving clonal architecture in Myeloma. Blood. 2015;126(23):21.

31. Hervé $\mathrm{M}$, et al. Unmutated and mutated chronic lymphocytic leukemias derive from self-reactive B cell precursors despite 
expressing different antibody reactivity. J Clin Invest. 2005;115 (6):1636-1643.

32. Mistry PK, et al. Effect of oral eliglustat on splenomegaly in patients with Gaucher disease type 1: the ENGAGE randomized clinical trial. JAMA. 2015;313 (7):695-706.

33. Tiller T, Meffre E, Yurasov S, Tsuiji M, Nussenzweig MC, Wardemann H. Efficient generation of monoclonal antibodies from single human B cells by single cell RT-PCR and expression vector cloning. J Immunol Methods. 2008;329 (1-2):112-124.

34. von Boehmer L, et al. Sequencing and cloning of antigen-specific antibodies from mouse memory B cells. Nat Protoc. 2016;11(10):1908-1923.

35. Testerink C, Larsen PB, van der Does D, van Himbergen JA, Munnik T. Phosphatidic acid binds to and inhibits the activity of Arabidopsis CTR1. J Exp Bot. 2007;58 (14):3905-3914.

36. Yeung T, Gilbert GE, Shi J, Silvius J, Kapus A, Grinstein S. Membrane phosphatidylserine regulates surface charge and protein localization. Science. 2008;319 (5860):210-213.

37. Bazzi MD, Nelsestuen GL. Extensive segregation of acidic phospholipids in membranes induced by protein kinase C and related proteins. Biochemistry. 1991;30 (32):7961-7969.

38. Shevchenko A, Tomas H, Havlis J, Olsen JV, Mann M. In-gel digestion for mass spectrometric characterization of proteins and proteomes. Nat Protoc. 2006;1 (6):2856-2860.

39. Keller A, Nesvizhskii AI, Kolker E, Aebersold R. Empirical statistical model to estimate the accuracy of peptide identifications made by MS/MS and database search. Anal Chem. 2002;74 (20):5383-5392.

40. Nesvizhskii AI, Keller A, Kolker E, Aebersold R. A statistical model for identifying proteins by tandem mass spectrometry. Anal Chem. 2003;75 (17):4646-4658. 